\title{
OBSERVATIONAL LIMITS ON COMPANIONS TO G29-38
}

\author{
S. J. Kleinman, ${ }^{1,2}$ R. E. Nather, ${ }^{1}$ D. E. Winget, ${ }^{1}$ J. C. Clemens, ${ }^{1,3,4}$ P. A. Bradley, ${ }^{1,5}$ A. KanaAn, ${ }^{1,6}$ \\ J. L. Provencal, ${ }^{1,7,8}$ C. F. Claver, ${ }^{1}$ T. K. Watson, ${ }^{1}$ K. Yanagida, ${ }^{1,9}$ J. S. Dixson, ${ }^{1}$ M. A. Wood, ${ }^{10,11}$ \\ D. J. Sullivan, ${ }^{12}$ E. Meištas, ${ }^{13,14}$ E. M. Leibowitz, ${ }^{15}$ P. Moskalik, ${ }^{16}$ S. Zola, ${ }^{17}$ G. Pajdosz, ${ }^{18}$ \\ J. Krzesinski, ${ }^{18}$ J.-E. Solheim, ${ }^{14,19}$ A. Bruvold, ${ }^{19}$ D. O’Donoghue, ${ }^{20}$ M. KATZ, ${ }^{20}$ G. VAuClair, ${ }^{21}$ \\ N. Dolez, ${ }^{21}$ M. Chevreton, ${ }^{22}$ M. A. Barstow, ${ }^{23}$ S. O. KePler, ${ }^{24}$ O. Giovannini, ${ }^{24,25}$ \\ C. J. HANSEN, ${ }^{26}$ AND S. D. KAWALER ${ }^{3}$ \\ Received 1994 January 31 ; accepted 1994 June 6
}

\begin{abstract}
Recent interest in the variable (DAV) white dwarf G29-38 has been stirred by a tentative report of a radial velocity variation that may be due to an unseen companion. Earlier evidence for a brown dwarf in the system has come from an observed infrared excess in the star's spectrum. For asteroseismological reasons, we have accumulated more than five seasons of high-speed photometric data on the star. By measuring the phase of an isolated, stable frequency in the power spectrum, we show the measured variation is not due (at least in its entirety) to an orbital companion. Because any orbital radial velocity variation must result in a systematic phase variation, the data we present can be used to place stringent limits on the types of companions the system may contain.
\end{abstract}

Subject headings: binaries: general - stars: individual (G29-38) - stars: low-mass, brown dwarfs stars: oscillations - white dwarfs

\footnotetext{
${ }^{1}$ Astronomy Department, University of Texas, Austin, TX 78712.

2 Guest Observer, Mount Stromlo and Siding Observatory, N.S.W., Australia.

${ }^{3}$ Current postal address: Department of Physics and Astronomy, Iowa State University, Ames, IA 50211.

${ }^{4}$ Hubble Fellow.

${ }^{5}$ Current postal address: X-2, MS B-220, Los Alamos National Laboratory, Los Alamos, NM 87545.

${ }^{6}$ Guest Observer, Isaac Newton Telescope, Roque de los Muchachos, La Palma, Canaries.

${ }^{7}$ Current postal address: University of Delaware, Physics and Astronomy Department, Sharp Laboratory, Newark, DE 19716.

${ }^{8}$ Guest Observer, Cerro Tololo Inter-American Observatory, Chile.

${ }^{9}$ Current postal address: 5-35-11 Hongōdai, Sakae-ku Yokohama 247,
} Japan.

${ }^{10}$ Department of Physics and Space Sciences, Florida Institute of Technology, 150 West University Boulevard, Melbourne, FL 32901.

${ }_{11}$ Guest Observer, Institute for Astronomy, Honolulu, HI.

12 Department of Physics, Victoria University, Box 600 Wellington, New Zealand.

${ }^{13}$ Institute of Theoretical Physics and Astronomy, Goštauto 12, Vilnius 2600 , Lithuania.

${ }^{14}$ Guest Observer, Maidanak Observatory, Uzbekistan.

15 Department of Physics and Astronomy, University of Tel Aviv, Ramat Aviv, Tel Aviv 69978, Israel.

${ }_{16}$ Copernicus Astronomical Center, Warsaw, Poland.

${ }^{17}$ Astronomical Observatory, Jagiellonian University, ul. Orla 171, 30-244 Cracow, Poland.

${ }^{18}$ Mount Suhora Observatory, Cracow Pedagogical University, ul. Podchorążych 2, 30-084 Cracow, Poland.

${ }^{19}$ Institutt for Matematiske Realfag, Universitet i Tromso, 9000 Tromso, Norway.

${ }^{20}$ Department of Astronomy, University of Capetown, Rondebosch 7700, Cape Province, South Africa.

${ }^{21}$ Observatoire Midi-Pyrenees, 14 Avenue E. Belin, 31400 Toulouse, France.

${ }^{22}$ Observatoire de Paris-Meudon, F-92195 Meudon, Principal Cedex, France.

${ }^{23}$ Physics Department, University of Leicester, Leicester LE1 7RH, UK.

${ }^{24}$ Instituto de Fisica, Universidade Federal do Rio Grande do Sul, 91501-970 Porto Alegre-RS, Brasil.

${ }_{25}$ Visiting Astronomer, Laboratorio Nacional de Astrofisica, CNPq, Brasil.

${ }^{26}$ Joint Institute for Laboratory Astrophysics, University of Colorado, Box 440 , Boulder, CO 80309.

\section{INTRODUCTION}

G29-38 (Giclas 29-28, ZZ Psc, WD 2326+049, EG 159, LTT 16907) is a large-amplitude DAV (ZZ Ceti) pulsator whose variability was first reported by Shulov \& Kopatskaya (1974) and confirmed by McGraw \& Robinson (1975). The DAV stars are otherwise normal white dwarfs (Winget 1988) that show intrinsic luminosity variations with amplitudes as high as $30 \%$ and periods between 100 and $1000 \mathrm{~s}$. The field of asteroseismology (see Winget 1988, for example) uses the observed spectrum of pulsations to probe the stellar interior. While we have made much progress on some of the pulsating white dwarfs (Winget 1991; Clemens 1994), the more complicated, large-amplitude DAV pulsators have proven more difficult to understand.

G29-38's power spectrum varies on timescales of weeks to years and is typically dominated by a single large mode, although many lower amplitude modes also exist. The larger amplitude modes show complex, variable behavior typical of other large-amplitude DAVs. Existing amidst these dominant yet variable modes are a few regions with less power that show considerably more stability by remaining in the light curve while the larger modes come and go. The large-amplitude DAVs, in general, distinguish themselves from their lower amplitude cousins by their cooler temperatures, their longer dominant periodicities, and the presence of many peaks with frequencies that are linear combinations of other modes present in the star. Although the lower-amplitude DAVs are much more stable, we generally observe only a few pulsation modes, making their power spectra simpler, but nonetheless, difficult to interpret. The key to asteroseismology lies in identifying as many modes as possible - each mode probing a slightly different region of the stellar interior. We have therefore observed G29-38 through many seasons, observing many of its changing states and adding to the total number of observed pulsation modes. Our interest in the star is primarily in understanding its spectrum of pulsation modes as a means 
of exploring its internal structure through asteroseismology. As a by-product of our methods, however, we can also constrain its external environment to considerable precision.

Zuckerman \& Becklin (1987) found an infrared excess at wavelengths above $2 \mu \mathrm{m}$ in the star's spectrum. Two principal models have been proposed to explain this excess (Zuckerman \& Becklin 1987; Greenstein 1988; Liebert, Saffer, \& Pilachowski 1989; Graham et al. 1990a; Telesco et al. 1990; Tokunaga, Becklin, \& Zuckerman 1990): a near-by dust cloud or a brown dwarf companion. (See Zuckerman 1993 for a recent review of the infrared excess and its possible origin.) The Whole Earth Telescope (WET; Nather et al. 1990) observed this star in 1988 November (Winget et al. 1990) and reported an unexplained phase change of the largest amplitude oscillation, at first attributed to the presence of an unseen massive companion. By monitoring the phase, or time of arrival, of periodic signals (in the form of stellar pulsations) from the star, Winget et al. looked for the systematic changes a binary orbit would produce. While in orbit, periodic signals from the star will have their phases delayed and advanced due to the continuously changing line-of-sight distance between the observer and the star. Simply put, when the star is farther away, light takes longer to get here; when it's closer, light arrives sooner. As distinct from measuring instantaneous radial velocities, this is an integral technique and, therefore, more sensitive. A low radial-velocity orbit can easily be detected given enough time and a stable isolated frequency in the power spectrum.

The dominant peak in Winget et al.'s data showed a significant phase variation, but since all periodic signals (pulsations) from the star must show precisely the same phase modulation if its cause is reflex orbital motion, they looked for another peak whose phase they could measure. They found a second stable peak with a period near $284 \mathrm{~s}$ that did not show the large phase modulation seen in the dominant mode - effectively eliminating the binary model (Winget et al. 1990; Kleinman 1990). Radial velocity measurements (Graham et al. 1990b) agreed with the photometric results and showed there was no massive companion in the system. Because these observations extended over only a few months of one season, the question of the existence of a less massive companion remained open.

More recently, Barnbaum \& Zuckerman (1992) found evidence for a small radial velocity variation of $\approx 10 \mathrm{~km} \mathrm{~s}^{-1}$ and a period of 11.2 months. They suggest the variation may be caused by an orbiting, low-mass companion-an attractive idea given the observed infrared excess, although the uncertainties in their data preclude making a definitive statement at this time. Given the ambiguity of the proposed system inclination, other types of companions are also possible. Using the data we have collected to answer asteroseismological questions about G29-38, we have analyzed the arrival times of the 284 second pulsation and have found no evidence of any orbital motion in this system that would yield measurable radial velocity variations.

\section{OBSERVATIONS}

Using high-speed photomultiplier-based photometers and observing (and reducing) procedures as described in Nather et al. (1990), we observed G29-38 from various sites over a 5 year time span. Table 1 lists the date, time, and location of each run. The data are primarily single-site data taken at the 36 inch (91 $\mathrm{cm})$ telescope at McDonald observatory supplemented by WET data during 1988 November (WET II: principal target; Winget et al. 1990) and 1992 September (WET VIII: low- priority target). With the exception of the runs from South Africa during the WET II campaign, the data consist of at least two channels-one watching the variable star and the other monitoring conditions by observing a nearby comparison star. Many of the McDonald runs, and some of the other WET runs, were taken with a three-channel instrument-the third channel measuring sky brightness continuously. Observers with less than three channels interrupted the data at irregular, but frequent, intervals to measure sky brightness as conditions warranted. The integration times were typically 5 or $10 \mathrm{~s}$ and were left unsummed in the final analysis.

For data taken nightly from a single site, a pattern of aliases separated by a frequency interval of one cycle per day arises due to the uncertainty in determining the cycle count during the gaps in the data. WET observations, which attempt to obtain as complete coverage as possible, eliminate or minimize the pattern of one cycle per day aliases, as demonstrated in Nather et al. (1990). The present data set is a combination of single-site data with WET data and so suffers from the presence of aliases, but at a reduced level because of the inclusion of the WET data. The spectral windows plotted in Figure 1 clearly demonstrate this point. (WET data on G29-38 was obtained during 1988 and 1992.)

\section{THE 284 SECOND PULSATION}

G29-38's power spectrum changes dramatically on timescales of order months or less-finding a well-defined mode present throughout the entire data set is difficult. Figure 1 shows a portion of the Fourier transform of the data taken in each individual observing season (1988-1992). There are only two identified regions of power that remain relatively constant. These two regions are at 2500 and $3500 \mu \mathrm{Hz}$. The $2500 \mu \mathrm{Hz}$ region is actually a multiplet of modes, with varying power in each component. The $3500 \mu \mathrm{Hz}$ mode (its actual frequency is near $3523 \mu \mathrm{Hz}$ corresponding to a period near $284 \mathrm{~s}$ ), however, has no nearby power and is well isolated in each data set. Its amplitude has remained relatively constant, always near 5 mmas. We now choose to represent amplitudes of modes as seen in a Fourier transform, by the unit mma, for milimodulation amplitude. It is similar to the traditional stellar luminosity measurement of magnitudes, in that $1 \mathrm{mma} \approx 1$ mmag, but it employs a linear, rather than logarithmic scale. Figure 2 is an enlargement of the Fourier transforms around this mode. Figure 3 shows a much smaller region of the transform at increased resolution for the entire (5 year) data set. Plotted with the transforms in the spectral window, obtained by taking the transform of a single noise-free sine curve sampled in the same way as the original data. The window represents the pattern of peaks a single sinusoidal variation will make in the transform. The improved windows of 1988 and 1992 are due to the single-site data being supplemented by WET data. Starting in 1990 , there is power near the $284 \mathrm{~s}$ mode that was not present in previous years. This additional power is harmonically related to a larger amplitude, lower frequency peak that appeared at the same time and is easily resolvable from the $284 \mathrm{~s}$ peak with the data we have. The $284 \mathrm{~s}$ mode is stable and isolated and thus well suited for phase analysis.

Since the data span such a large timebase, we must have an accurate period in order to avoid cycle count errors when measuring pulsation arrival times. Cycle count errors arise when the uncertainty in period is large enough that the number of pulses that occurred through a gap in the data is ambiguous. With such intense coverage, though, this potential stumbling 
TABLE 1

TABLE OF OBSERVATIONS

\begin{tabular}{|c|c|c|c|c|}
\hline Telescope & Run Name & $\begin{array}{l}\text { Date } \\
\text { (UT) }\end{array}$ & Runstart & Group \\
\hline McDonald 30" & jcc- 55 & 1988 Oct 24 & $2: 00: 00$ & - \\
\hline McDonald 30" & jec- 57 & 1988 Oct 25 & 1:50:00 & - \\
\hline McDonald 30" & jcc-59 & 1988 Oct 26 & $4: 50: 00$ & - \\
\hline $\mathrm{SAAO} 0.75 \mathrm{~m}$ & s4453 & 1988 Nov 4 & $18: 38: 0$ & 1 \\
\hline $\mathrm{SAAO} 0.75 \mathrm{~m}$ & s4455 & 1988 Nov 6 & $18: 23: 0$ & 1 \\
\hline McDonald 82" & maw-0017 & 1988 Nov 6 & $1: 42: 20$ & 1 \\
\hline McDonald 82" & maw-0019 & 1988 Nov 7 & $1: 31: 00$ & 1 \\
\hline SidingSpring $40 "$ & ren-0040 & 1988 Nov 7 & $11: 12: 30$ & 1 \\
\hline SidingSpring $40 "$ & ren-0042 & 1988 Nov 8 & $9: 41: 00$ & 1 \\
\hline McDonald 82" & maw-0023 & 1988 Nov 9 & $1: 21: 10$ & 1 \\
\hline McDonald 82" & maw-0024 & 1988 Nov 9 & $4: 54: 40$ & 1 \\
\hline SidingSpring $40 "$ & ren-0044 & 1988 Nov 9 & $9: 41: 10$ & 1 \\
\hline SAAO $0.75 \mathrm{~m}$ & $\mathrm{~s} 4456$ & 1988 Nov 9 & $18: 53: 3$ & 1 \\
\hline McDonald 82" & maw-0026 & 1988 Nov 10 & $1: 23: 00$ & 2 \\
\hline SidingSpring $40 "$ & ren-0045 & 1988 Nov 10 & $9: 28: 50$ & 2 \\
\hline SAAO $0.75 \mathrm{~m}$ & s4457 & 1988 Nov 10 & $18: 33: 5$ & 2 \\
\hline McDonald 36" & cfc-0001 & 1988 Nov 11 & $1: 51: 00$ & 2 \\
\hline OHP $1.93 \mathrm{~m}$ & gv37 & 1988 Nov 11 & 18:59:20 & 2 \\
\hline McDonald 82" & maw-0029 & 1988 Nov 12 & $1: 24: 10$ & 2 \\
\hline McDonald 36" & cfc- -0002 & 1988 Nov 12 & $3: 41: 01$ & 2 \\
\hline MaunaKea 24" & a91 & 1988 Nov 12 & $5: 12: 00$ & 2 \\
\hline SidingSpring 40" & ren-0049 & 1988 Nov 12 & 9:30:10 & 2 \\
\hline $\mathrm{SAAO} 0.75 \mathrm{~m}$ & $\mathrm{~s} 4458 \mathrm{a}$ & 1988 Nov 12 & $19: 41: 5$ & 2 \\
\hline McDonald 82" & maw-0031 & 1988 Nov 13 & $0: 51: 20$ & 2 \\
\hline McDonald 36" & cfc-0005 & 1988 Nov 13 & $3: 03: 50$ & 2 \\
\hline MaunaKea 24" & a93 & 1988 Nov 13 & $8: 04: 00$ & 2 \\
\hline SidingSpring $40 "$ & ren-0053 & 1988 Nov 13 & $9: 28: 20$ & 3 \\
\hline OHP $1.93 \mathrm{~m}$ & gv39 & 1988 Nov 13 & $18: 12: 30$ & 3 \\
\hline McDonald 82" & maw-0033 & 1988 Nov 14 & $1: 04: 00$ & 3 \\
\hline McDonald 36" & cfc-0009 & 1988 Nov 14 & $3: 22: 30$ & 3 \\
\hline McDonald $36 "$ & cfc-0010 & 1988 Nov $1^{\prime} 5$ & $3: 46: 30$ & 3 \\
\hline McDonald 82" & maw-0036 & 1988 Nov 16 & $1: 07: 20$ & 3 \\
\hline MaunaKea 24" & a97 & 1988 Nov 16 & $6: 29: 00$ & 3 \\
\hline McDonald 82" & maw-0038 & 1988 Nov 17 & $0: 58: 20$ & 3 \\
\hline McDonald 82" & maw-0039 & 1988 Nov 17 & $2: 47: 20$ & 3 \\
\hline $\mathrm{SAAO} 0.75 \mathrm{~m}$ & s4466 & 1988 Nov 17 & $18: 43: 0$ & 3 \\
\hline McDonald 82" & maw-0040 & 1988 Nov 18 & $6: 05: 40$ & 3 \\
\hline SAAO $0.75 \mathrm{~m}$ & s4468 & 1988 Nov 18 & $18: 43: 0$ & 3 \\
\hline McDonald 82" & maw-0042 & 1988 Nov 19 & $0: 52: 20$ & 3 \\
\hline McDonald 30" & mlf-0001 & 1988 Nov 20 & $1: 19: 28$ & 3 \\
\hline $\mathrm{SAAO} 0.75 \mathrm{~m}$ & $\mathrm{~s} 4472 \mathrm{a}$ & 1988 Nov 21 & $18: 52: 0$ & 3 \\
\hline McDonald 30" & mlf-0003 & 1988 Nov 22 & $1: 04: 03$ & 4 \\
\hline McDonald 30" & sjk-0004 & 1988 Nov 23 & $1: 42: 50$ & 4 \\
\hline McDonald 30" & sjk-0005 & 1988 Nov 24 & $1: 29: 40$ & 4 \\
\hline McDonald 30" & cfc-0016 & 1988 Nov 26 & $1: 01: 40$ & 4 \\
\hline McDonald 30" & $\mathrm{cfc}-0017$ & 1988 Nov 27 & $0: 50: 00$ & 4 \\
\hline McDonald 30" & cfc-0018 & 1988 Nov 28 & $0: 57: 50$ & 4 \\
\hline $\mathrm{SAAO} 0.75 \mathrm{~m}$ & s4480 & 1988 Nov 28 & $19: 29: 1$ & 5 \\
\hline McDonald 30" & pab-0002 & 1988 Nov 29 & $1: 14: 50$ & 5 \\
\hline McDonald 36" & pab-0003 & 1988 Nov 29 & $3: 24: 20$ & 5 \\
\hline McDonald 36" & pab-0004 & 1988 Dec 1 & $0: 44: 30$ & 5 \\
\hline MaunaKea CFHT & jcc-0063 & 1988 Dec 1 & $5: 54: 30$ & 5 \\
\hline MaunaKea CFHT & jcc-0066 & 1988 Dec 2 & 4:59:10 & 5 \\
\hline McDonald 36" & pab-0009 & 1988 Dec 3 & $0: 54: 10$ & 5 \\
\hline McDonald 36" & pab-0011 & 1988 Dec 4 & $0: 56: 20$ & 5 \\
\hline McDonald 36" & mlf-0004 & 1988 Dec 14 & $1: 21: 21$ & - \\
\hline McDonald 36" & mlf-0005 & $1988 \operatorname{Dec} 15$ & $1: 17: 04$ & - \\
\hline McDonald 36" & jcc-0069 & 1988 Dec 21 & $1: 25: 00$ & - \\
\hline McDonald 36" & jcc-0071 & 1988 Dec 22 & $1: 24: 00$ & - \\
\hline KPNO 1.3-m & a100 & $1988 \mathrm{Dec} 30$ & $2: 47: 00$ & - \\
\hline KPNO $1.3-\mathrm{m}$ & a104 & 1988 Dec 31 & $1: 57: 00$ & - \\
\hline KPNO $1.3-\mathrm{m}$ & a107 & $1989 \operatorname{Jan} 1$ & $1: 57: 00$ & . \\
\hline
\end{tabular}


TABLE 1-Continued

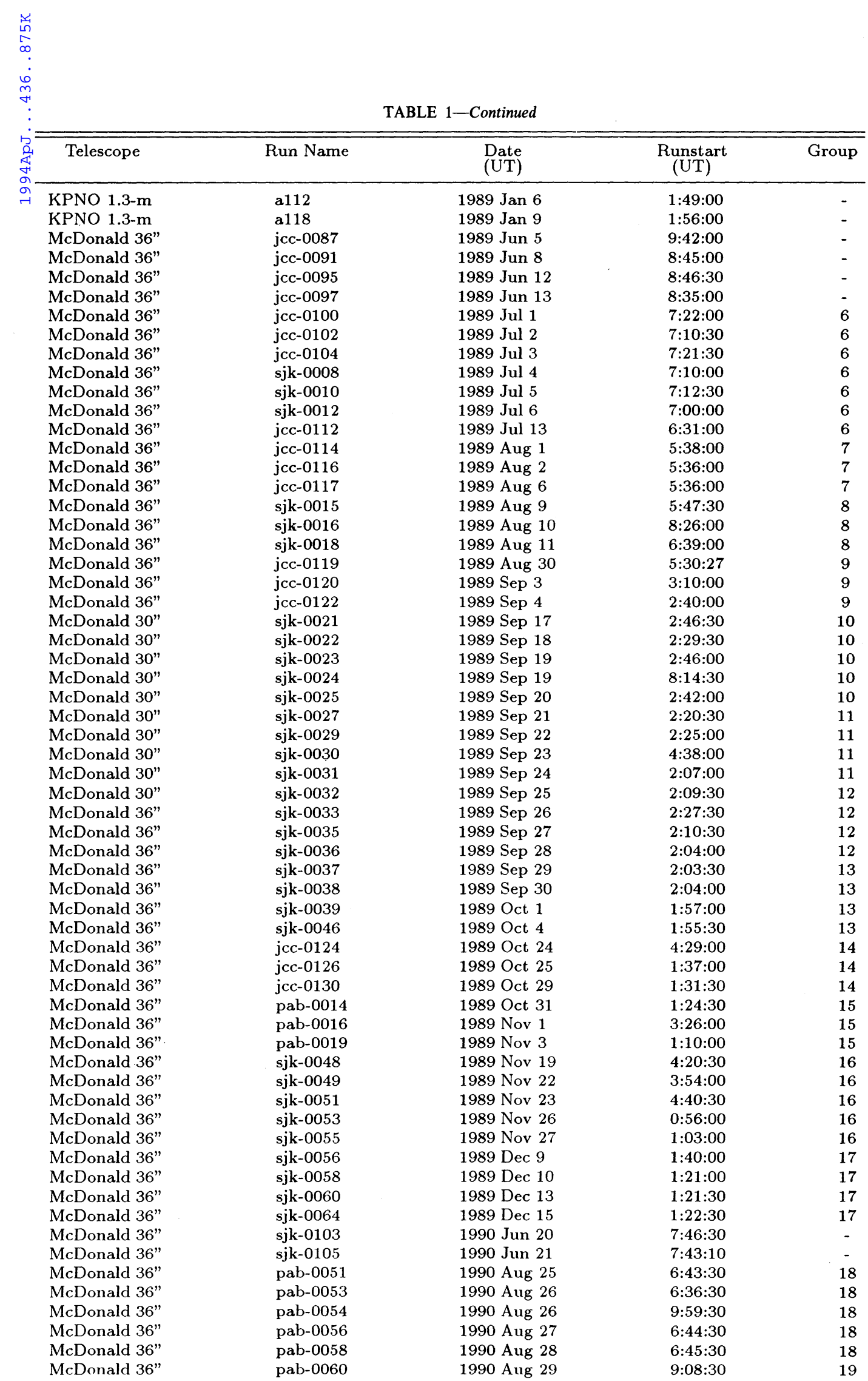


TABLE 1-Coníinued

\begin{tabular}{|c|c|c|c|c|}
\hline Telescope & Run Name & $\begin{array}{l}\text { Date } \\
\text { (UT) }\end{array}$ & $\underset{\text { (UT) }}{\text { Runstart }}$ & Group \\
\hline McDonald 36" & pab-0062 & 1990 Aug 30 & $5: 51: 30$ & 19 \\
\hline McDonald 36" & pab-0064 & 1990 Aug 31 & $5: 26: 00$ & 19 \\
\hline McDonald 30" & pab-0065 & 1990 Sep 1 & $5: 01: 00$ & 19 \\
\hline McDonald 36" & sjk-0106 & 1990 Sep 9 & $6: 58: 00$ & 20 \\
\hline McDonald 36" & sjk-0107 & 1990 Sep 10 & $2: 28: 30$ & 20 \\
\hline McDonald 36" & sjk-0108 & 1990 Sep 11 & $2: 37: 30$ & 20 \\
\hline McDonald 36" & sjk-0109 & 1990 Sep 12 & $2: 28: 30$ & 20 \\
\hline McDonald 36" & sjk-0110 & 1990 Sep 18 & $3: 55: 00$ & 20 \\
\hline McDonald 36" & jcc-0159 & 1990 Sep 28 & $3: 40: 30$ & - \\
\hline McDonald 36" & jcc-0163 & 1990 Oct 9 & 1:50:00 & 21 \\
\hline McDonald 36" & jcc-0164 & 1990 Oct 10 & $2: 01: 00$ & 21 \\
\hline McDonald 36" & jcc-0166 & 1990 Oct 11 & $2: 32: 00$ & 21 \\
\hline McDonald 82" & g293020 & 1990 Oct 20 & $1: 59: 28$ & 21 \\
\hline McDonald 82" & g293027 & 1990 Oct 22 & $1: 46: 13$ & 21 \\
\hline McDonald 82" & g293034 & 1990 Oct 23 & 1:40:29 & 21 \\
\hline McDonald 36" & sjk-0112 & 1990 Oct 24 & $2: 22: 30$ & 21 \\
\hline McDonald 36" & sjk-0114 & 1990 Oct 25 & $1: 20: 00$ & 21 \\
\hline McDonald 36" & sjk-0116 & 1990 Oct 26 & $1: 22: 30$ & 21 \\
\hline McDonald 36" & sjk-0118 & 1990 Oct 27 & $1: 14: 00$ & 21 \\
\hline McDonald 36" & sjk-0121 & 1990 Oct 29 & $1: 15: 00$ & 21 \\
\hline McDonald 36" & sjk-0123 & 1990 Nov 10 & $1: 08: 00$ & 22 \\
\hline McDonald 36" & sjk-0125 & 1990 Nov 11 & 1:04:00 & 22 \\
\hline McDonald 36" & sjk-0129 & 1990 Nov 14 & $1: 04: 30$ & 22 \\
\hline McDonald 36" & sjk-0130 & 1990 Dec 18 & $2: 55: 00$ & - \\
\hline McDonald 36" & sjk-0132 & 1990 Dec 19 & $1: 21: 30$ & - \\
\hline McDonald 36" & sjk-0134 & 1990 Dec 20 & $2: 24: 00$ & - \\
\hline McDonald 36" & sjk-0136 & 1990 Dec 21 & $3: 02: 30$ & - \\
\hline McDonald 36" & sjk-0143 & 1991 Jan 16 & 1:19:00 & - \\
\hline McDonald 36" & jcc-0185 & 1991 Jul 18 & $7: 44: 00$ & - \\
\hline McDonald 36" & sjk-0156 & 1991 Sep 4 & $3: 01: 00$ & - \\
\hline McDonald 36" & sjk-0158 & 1991 Sep 8 & $7: 38: 00$ & - \\
\hline McDonald 82" & jcc- 188 & 1991 Oct 16 & $1: 47: 00$ & 23 \\
\hline McDonald 82" & jec-190 & 1991 Oct 17 & $1: 46: 00$ & 23 \\
\hline McDonald 36" & sjk-0164 & 1991 Oct 17 & $3: 11: 00$ & 23 \\
\hline McDonald 36" & sjk-0166 & 1991 Nov 4 & $1: 51: 00$ & 24 \\
\hline McDonald 36" & sjk-0168 & 1991 Nov 5 & $1: 51: 30$ & 24 \\
\hline McDonald 36" & sjk-0171 & 1991 Nov 6 & $1: 28: 00$ & 24 \\
\hline McDonald 36" & sjk-0173 & 1991 Nov 7 & $1: 27: 30$ & 24 \\
\hline McDonald 36" & sjk-0175 & 1991 Nov 9 & $1: 29: 00$ & 24 \\
\hline McDonald 36" & sjk-0177 & 1991 Nov 10 & $1: 25: 00$ & 24 \\
\hline McDonald 36" & sjk-0179 & 1991 Dec 11 & $2: 31: 00$ & 25 \\
\hline McDonald 36" & sjk-0180 & 1991 Dec 13 & $2: 09: 00$ & 25 \\
\hline McDonald 36" & sjk-0182 & 1991 Dec 14 & $1: 22: 00$ & 25 \\
\hline McDonald 82" & tkw-0017 & 1992 Aug 23 & $9: 13: 30$ & - \\
\hline LNA $1.6 \mathrm{~m}$ & ro018 & 1992 Sep 22 & $3: 24: 30$ & 26 \\
\hline LaPalma INT & int-0009 & 1992 Sep 22 & $3: 54: 40$ & 26 \\
\hline LaPalma INT & int-0013 & 1992 Sep 23 & $2: 42: 50$ & 26 \\
\hline CTIO $1.5 \mathrm{~m}$ & jlp-0114 & 1992 Sep 23 & $6: 25: 45$ & 26 \\
\hline CTIO $1.5 \mathrm{~m}$ & jlp-0117 & 1992 Sep 24 & $6: 28: 35$ & 26 \\
\hline Maidanak $1.0 \mathrm{~m}$ & jesem-07 & 1992 Sep 24 & $21: 51: 00$ & 26 \\
\hline LaPalma INT & int-0016 & 1992 Sep 25 & $2: 09: 10$ & 26 \\
\hline McDonald 82" & pab-0154 & 1992 Sep 25 & $9: 26: 00$ & 26 \\
\hline Maidanak 1.0m & jesem-09 & 1992 Sep 25 & $20: 53: 20$ & 26 \\
\hline CTIO $1.5 \mathrm{~m}$ & jlp-0122 & 1992 Sep 26 & $6: 30: 10$ & 27 \\
\hline McDonald 82" & pab-0158 & 1992 Sep 26 & $9: 26: 00$ & 27 \\
\hline OHP $1.93 \mathrm{~m}$ & $\mathrm{gv}-0236$ & 1992 Sep 26 & $1: 56: 30$ & 27 \\
\hline CTIO $1.5 \mathrm{~m}$ & jlp-0124 & 1992 Sep 27 & $6: 31: 40$ & 27 \\
\hline McDonald 82" & pab-0161 & 1992 Sep 27 & $9: 55: 30$ & 27 \\
\hline Maidanak $1.0 \mathrm{~m}$ & jesem-13 & 1992 Sep 27 & $22: 49: 50$ & 27 \\
\hline LaPalma INT & int-0023 & 1992 Sep 28 & $3: 49: 50$ & 27 \\
\hline CTIO $1.5 \mathrm{~m}$ & jlp-0127 & 1992 Sep 28 & $6: 17: 00$ & 27 \\
\hline McDonald 82" & pab-0164 & 1992 Sep 28 & $10: 01: 30$ & 27 \\
\hline
\end{tabular}


TABLE 1-Continued

\begin{tabular}{clcrc}
\hline \hline Telescope & Run Name & $\begin{array}{c}\text { Date } \\
\text { (UT) }\end{array}$ & $\begin{array}{c}\text { Runstart } \\
\text { (UT) }\end{array}$ & Group \\
\hline McDonald 82" & pab-0167 & 1992 Sep 29 & $9: 48: 30$ & 27 \\
MaunaKea 24" & maw-0110 & 1992 Sep 29 & $12: 21: 10$ & 27 \\
OHP 1.93m & gv-0244 & 1992 Sep 29 & $23: 24: 40$ & 28 \\
McDonald 82" & pab-0169 & 1992 Sep 30 & $9: 45: 00$ & 28 \\
MaunaKea 24" & maw-0112 & 1992 Sep 30 & $12: 19: 20$ & 28 \\
SSO 40" & sjk-0209 & 1992 Sep 30 & $14: 39: 30$ & 28 \\
OHP 1.93m & gv-0247 & 1992 Sep 30 & $23: 52: 30$ & 28 \\
McDonald 82" & pab-0172 & 1992 Oct 1 & $9: 44: 00$ & $28: 41: 40$ \\
MaunaKea 24" & maw-0115 & 1992 Oct 1 & $9: 36: 30$ & 28 \\
McDonald 82" & pab-0174 & 1992 Oct 2 & $1: 05: 30$ & 29 \\
McDonald 36" & sjk-0220 & 1992 Oct 26 & $1: 42: 30$ & 29 \\
McDonald 36" & sjk-0223 & 1992 Oct 27 & $0: 45: 00$ & 30 \\
McDonald 36" & jlp-0130 & 1992 Nov 23 & $0: 38: 10$ & 30 \\
McDonald 36" & jlp-0133 & 1992 Nov 25 & $2: 09: 00$ & - \\
McDonald 36" & tkw-0019 & 1993 Jan 11 & $1: 26: 30$ & - \\
McDonald 36" & tkw-0021 & 1993 Jan 12 & $1: 09: 00$ & - \\
McDonald 36" & tkw-0025 & 1993 Jan 14 & & \\
& & & &
\end{tabular}

NoTE.- The location, name, and UT date and time are listed for each run analyzed. Runs with the same group number were joined together to obtain better timings. Runs without numbers were used to calculate the Fourier transforms, but were not suitable for timing measurements of the $284 \mathrm{~s}$ peak.

block was not a problem. The period can be determined either through the Fourier transform, or by a nonlinear least-squares fit to the data set of a single sinusoid of unknown period, phase, and amplitude. Since the nonlinear least-squares fit is basically a single-point Fourier transform, both methods should (and do) yield the same answer. The nonlinear leastsquares technique, however, has the advantage of providing a quantifiable uncertainty.

Starting with the WET II data, we were able to determine a period accurate enough to bridge the data gap to the next observing month and bridge our way up month by month and then year by year to cover the entire data set. The net result is a period of $283.866459 \pm 0.000007 \mathrm{~s}$-accurate enough to bridge a data gap of 37 years and still be certain of the cycle count. Table 2 lists the best measured period of each year. Given their uncertainties, the yearly periods are consistent with a constant period model, although there is a suggestion of a slightly decreasing period over time. In any case, the rate of change is small enough that the faster variation we are seeking here is certainly detectable.

Having determined an accurate period, we used it to calculate an ephemeris and predict the arrival times of the pulse maxima, or phase, of the $284 \mathrm{~s}$ period. The arrival times are calculated assuming a constant period and no phase change. To be consistent with the proposed binary model, we should

TABLE 2

MEASURED Period

\begin{tabular}{|c|c|}
\hline Year & $\begin{array}{l}\text { Period } \\
\text { (s) }\end{array}$ \\
\hline $1988 .$. & $283.8674 \pm 0.0008$ \\
\hline 1989. & $283.86681 \pm 0.00007$ \\
\hline $1990 .$. & $283.8667 \pm 0.0002$ \\
\hline .............. & $283.8666 \pm 0.0002$ \\
\hline 1992. & $283.8664 \pm 0.0002$ \\
\hline
\end{tabular}

Nore.-These are the periods for the $284 \mathrm{~s}$ pulsation as measured each year. The best fit measurement for the entire data set is $283^{\mathrm{s}} 866459 \pm 0.000007$. choose the phase zero point so the mean deviation of actual arrival times $(O$ : observed) from the predicted times $(C$ : calculated), the $(O-C)$, is zero.

After determining the pulsation ephemeris, we measured the phase in each of our data runs. Since this particular mode has a much lower amplitude than the star's dominant modes, and since there are sometimes other modes nearby that can interfere with it on short timescale, the individual runs in Table 1 were grouped to obtain a larger timebase, and hence a better phase measurement. The size of each group depended on the amplitude of the mode (it was a bit lower in 1988 than in the other years) and on the density of data. Runs were added together until a reasonable uncertainty was obtained. There is always a tradeoff between phase uncertainty and temporal resolution; we have tried to make the best choice. In each case, we carefully noted that the actual phase measurement did not change significantly while the uncertainty continued to decrease as the timebase (and hence, number of cycles) increased.

The group membership of each individual run is also listed in Table 1. Some otherwise fine observing runs did not yield enough data to allow an accurate measurement of this relatively low-amplitude peak. In such cases, the individual observing runs were used in the overall Fourier transforms, but not in the $(O-C)$ measurements. These runs are denoted by an " - " in the "Group" column of Table 1. We ran a linear leastsquares routine on each group to measure the phase and uncertainty of the $283^{\text {s.866459 }}$ pulsation. Having obtained the predicted and measured phase timings, we calculated the $(O-C)$ of the phase measurements and list them in Table 3. The phase zero point is arbitrary here. We have not yet tried to normalize it for consistency with the binary model. The errors we list in Table 3 are the $1 \sigma$ errors reported by the leastsquares fitting routine. This formal uncertainty, however, is always less than the actual scatter of the data. Simulations show that the increased scatter comes from two sources: noise in the data and the presence of other power at nearby frequencies. Since the errors we quote here are the fits' $1 \sigma$ errors, we must remember that these are typically underestimating the 


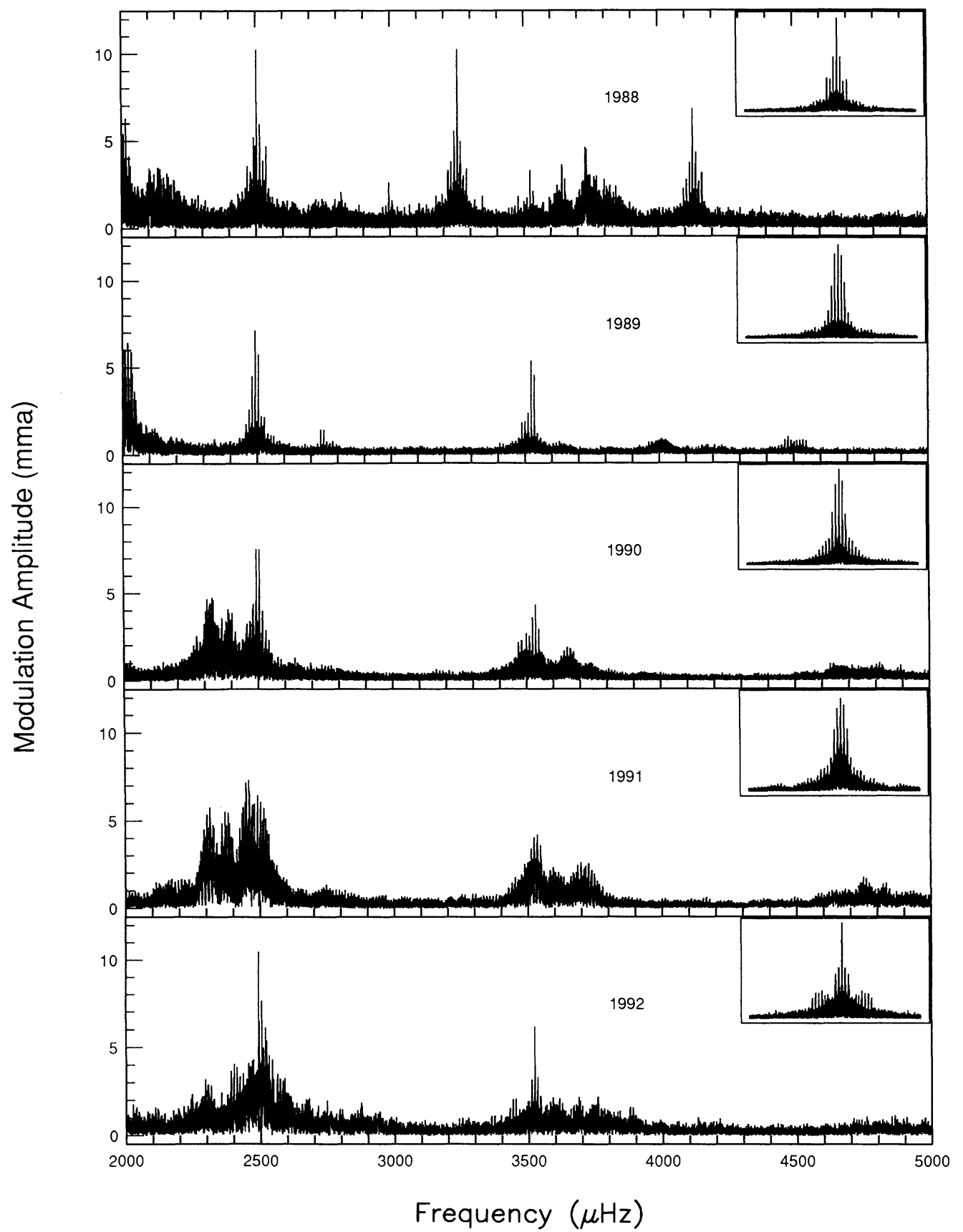

FIG. 1.-A portion of the FT from each year's data. The insert is the spectral window (described in text) obtained for each data set.

true uncertainty and use the actual scatter of the data as a better uncertainty indicator. The uncertainties in the 1988 data are typically larger due to the peak's lower amplitude that season.

\section{RESULTS}

The resultant $(O-C)$ diagram is shown in Figure 4. In the top panel of the figure are the observed radial velocity variations presented by Barnbaum \& Zuckerman (1992) and their proposed binary orbit fit. Using the parameters of their fit listed in Table 4, we calculated their predicted $(O-C)$ variation and plot it as the dashed line in the second panel along with our data listed in Table 3. $(O-C)$ phase normalization is unconstrained at this point since our data obviously do not match the prediction. Even if there is a longer period binary present in the system, we have observed less than a complete cycle and are unable to make a meaningful normalization. Our data could therefore be shifted along the $y$-axis by any arbitrary amount. The solid curve in the second panel is the best sinusoidal fit to our $(O-C)$ measurements which we do not claim is significant, but include for completeness. The plotted $(O-C)$ error bars are those determined by the $1 \sigma$ linear leastsquares fit. The timebase of both the radial velocity and phase measurements is relative to the run-start time of the first WET II run, s4453. The observed phase timings clearly contradict the proposed binary model. 


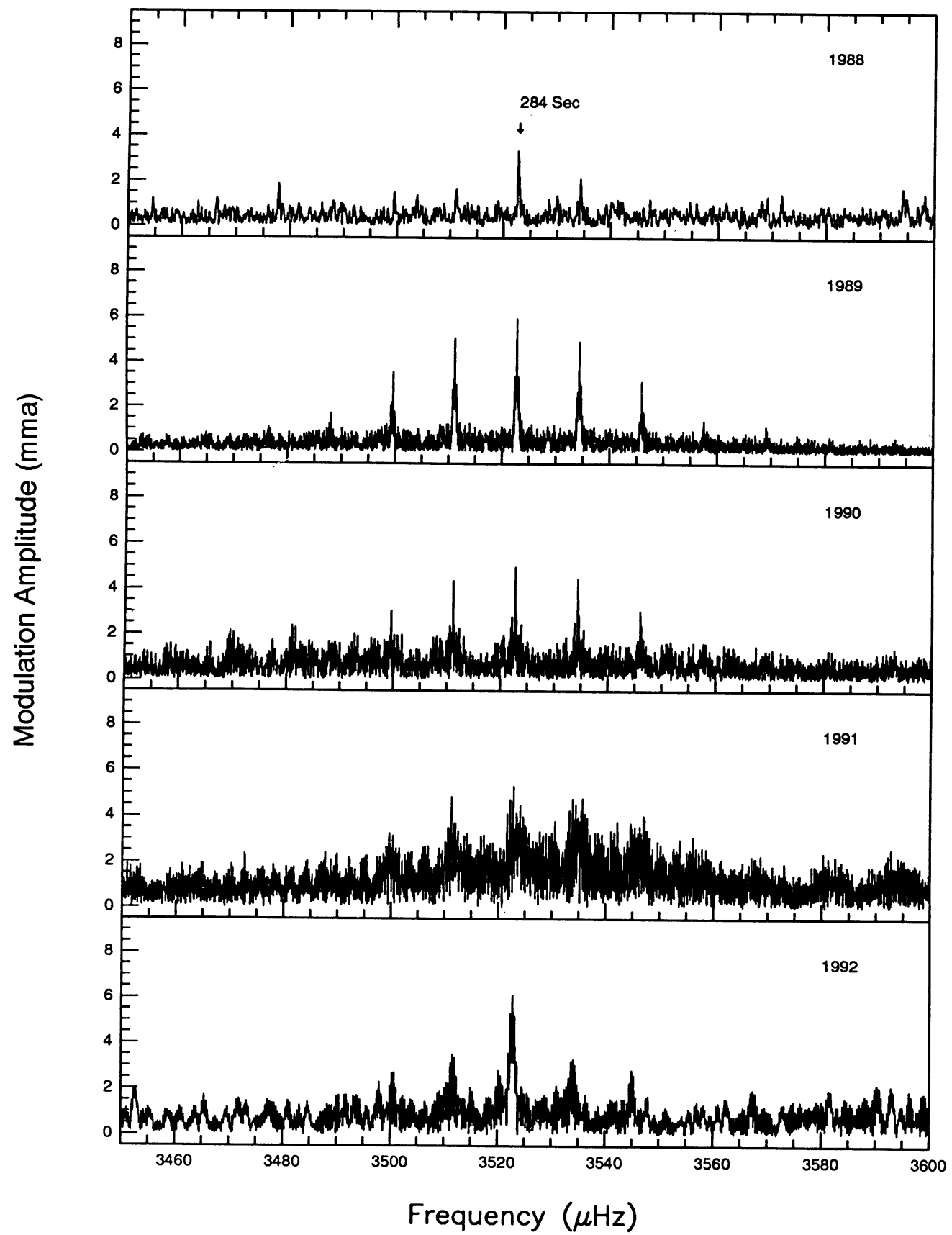

FIG. 2.-The FT from each year's data around the $284 \mathrm{~s}$ peak. Note the spectral window pattern (see Fig. 1) is clearly evident.

Although the proposed period is very close to one year, making complete phase coverage difficult, the $(O-C)$ data presented here represent nearly $75 \%$ phase coverage. The data from 1989 span 6 months and show no significant variation whereas the proposed model would have the phase varying over more than half an orbital cycle.

As a final test, we fit our data (now normalized to a mean of zero) to a sinusoid of the predicted period and added a linear term to accommodate any error in the pulsation's period determination. The best fit is a sine curve with amplitude $2 \pm 9 \mathrm{~s}$, nowhere near the required amplitude to match the radial velocity data and clearly indistinguishable from a straight line.

The observed trend in the $(O-C)$ diagram (and the best-fit periods of each year) may arise from a constant change in the pulsation period. Such changes are expected on asteroseismological grounds and can yield direct information about the star's evolution (see Kepler et al. 1991 for example). Kepler's result on another DAV, G117-B15A, is a rate of period change on the order of $10^{-15} \mathrm{~s} \mathrm{~s}^{-1}$. Our possible measurement is several orders of magnitude larger than that and is therefore not understandable with traditional evolutionary models. This question will be analyzed more carefully in a future publication.

It is also possible that the trend is due to reflex orbital motion with a rather long period, as our solid line sinusoidal fit in Figure 4 would imply. If so, the $56 \mathrm{~s}$ amplitude and $8.0 \mathrm{yr}$ 


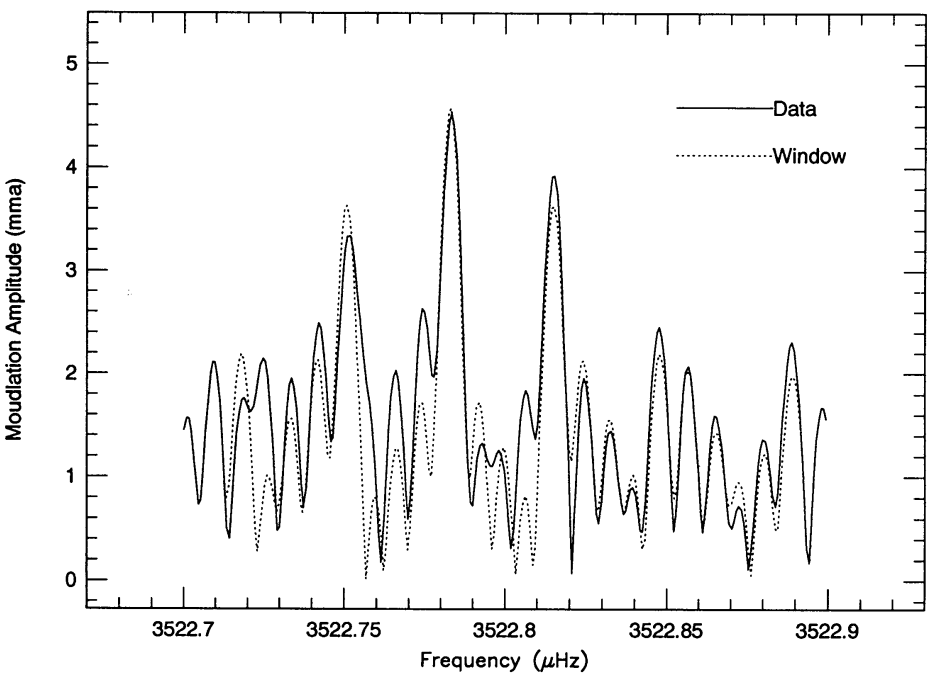

FIG. 3.-The FT and window of the entire data set near the $284 \mathrm{~s}$ peak

period of the fit would produce a peak-to-peak radial velocity variation of only $0.8 \mathrm{~km} \mathrm{~s}^{-1}-\mathrm{a}$ rather challenging measurement. Using the above amplitude and period, and assuming a mass of $0.6 M_{\odot}$ for $\mathrm{G} 29-38$, we can calculate a mass function and determine values of possible companion masses for varying orbital inclination angles. Note, however, the $56 \mathrm{~s}$ $(O-C)$ amplitude is related to the orbit of G29-38 itself, and is not the relative orbit of G29-38 to its companion. We must keep this in mind, and use the equation for the center of mass, when applying Kepler's equations to the problem. The wellknown form, therefore, of Kepler's third law is

$$
\frac{\left[a_{1} \sin (i)\right]^{3}}{P^{2}}=\frac{\left[M_{2} \sin (i)\right]^{3}}{\left(M_{1}+M_{2}\right)^{2}},
$$

where $P$ is the orbital period in years, $a_{1} \sin (i)$ is the measured $(O-C)$ amplitude converted to A.U.s (and is hence the projected semi-major axis of G29-38's orbit), $i$ is the inclination angle of the orbit, and $M_{1}$ and $M_{2}$ are the masses of G29-38 and the companion, respectively, in solar masses. The value of the lefthand side of the equation is $2.48 \times 10^{-5} M_{\odot}$, making the
TABLE 3

\begin{tabular}{|c|c|c|c|}
\hline Group & $\begin{array}{c}T_{\max } \\
\text { (s) }\end{array}$ & $\begin{array}{c}(O-C) \\
\text { (s) }\end{array}$ & $\begin{array}{c}\delta(O-C) \\
\text { (s) }\end{array}$ \\
\hline $1 \ldots$ & 11 & 0 & 7 \\
\hline (n............ & 456,467 & -1 & 9 \\
\hline $3 \ldots$ & 745,443 & -1 & 10 \\
\hline ............. & $1,492,017$ & 4 & 9 \\
\hline ….......... & $2,076,498$ & 4 & 9 \\
\hline …........... & $20,609,043$ & 43 & 2 \\
\hline ........ & $23,281,649$ & 46 & 4 \\
\hline .............. & $23,977,690$ & 47 & 4 \\
\hline $9 \ldots$ & $25,786,777$ & 53 & 4 \\
\hline …............ & $27,332,133$ & 40 & 2 \\
\hline $11 \ldots \ldots \ldots \ldots \ldots \ldots \ldots$ & $27,676,186$ & 47 & 2 \\
\hline ......... & $28,021,086$ & 49 & 2 \\
\hline n.............. & $28,366,265$ & 46 & 2 \\
\hline (.............. & $30,535,012$ & 54 & 3 \\
\hline $15 \ldots \ldots \ldots \ldots \ldots \ldots \ldots$ & $31,131,702$ & 56 & 5 \\
\hline $16 \ldots \ldots \ldots \ldots \ldots \ldots$ & $32,781,534$ & 57 & 5 \\
\hline ............. & $34,498,934$ & 65 & 4 \\
\hline n.............. & $56,895,132$ & 50 & 5 \\
\hline …............ & $57,249,401$ & 54 & 5 \\
\hline ............... & $58,192,113$ & 46 & 5 \\
\hline $21 \ldots \ldots+\ldots, \ldots+\ldots, \ldots$ & $60,765,378$ & 61 & 5 \\
\hline ............. & $63,527,673$ & 52 & 6 \\
\hline $23 \ldots \ldots \ldots \ldots \ldots \ldots \ldots$ & $92,906,170$ & 73 & 4 \\
\hline (............... & $94,547,758$ & 62 & 4 \\
\hline …........... & $97,746,631$ & 44 & 7 \\
\hline $26 \ldots \ldots \ldots \ldots \ldots \ldots \ldots$ & $122,460,580$ & 11 & 3 \\
\hline $27 \ldots \ldots \ldots \ldots \ldots \ldots \ldots$ & $122,817,409$ & 20 & 3 \\
\hline 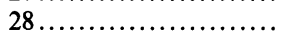 & $123,137,605$ & 14 & 3 \\
\hline …........... & $125,390,086$ & 15 & 5 \\
\hline $30 \ldots \ldots \ldots+\ldots$ & $127,807,776$ & 14 & 6 \\
\hline
\end{tabular}

Note.-The group number used in Table 1 is printed here along with the measured time of maximum of the $284 \mathrm{~s}$ pulsation. The timing residuals $(O-C)$ and their uncertainties are in the last two columns.

minimum mass of a companion $\sim 0.021 M_{\odot}$. For the companion to have a mass greater than $0.1 M_{\odot}$ would require an inclination of less than $13^{\circ}$. Let us remark again, however, that this result applies only if the observed trend in the $(O-C)$ diagram is due to reflex orbital motion and the current data set is simply not good enough to force us to this (or any other)

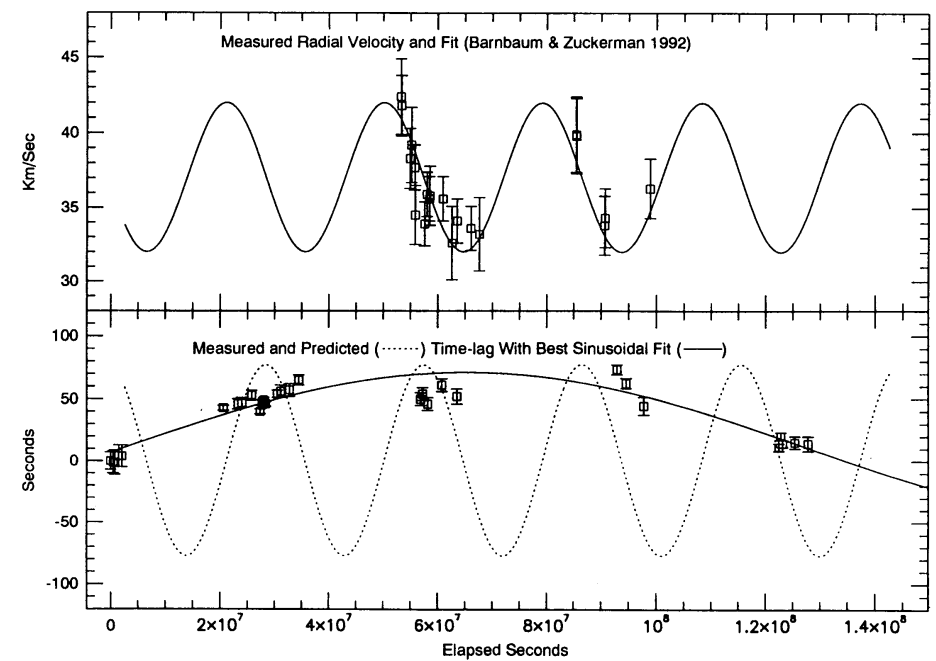

Fig. 4.-The measured and predicted radial velocity and $(O-C)$ values. The zero point of the $(O-C)$ points is arbitrary relative to the predicted curve (see text). 
TABLE 4

Parameters of Fit to Radial Velocity Variations

\begin{tabular}{cccc}
\hline $\begin{array}{c}\text { Amplitude } \\
\left(\mathrm{km} \mathrm{s}^{-1}\right)\end{array}$ & $\begin{array}{c}\text { Period } \\
(\mathrm{s})\end{array}$ & $\begin{array}{c}\text { Phase } \\
(\mathrm{s})\end{array}$ & $\begin{array}{c}\text { Offset } \\
\left(\mathrm{km} \mathrm{s}^{-1}\right)\end{array}$ \\
\hline $5.0 \ldots \ldots \ldots \ldots \ldots \ldots \ldots \ldots \ldots \ldots \ldots \ldots \ldots$ & $29 \times 10^{6}$ & $1.4 \times 10^{7}$ & 37 \\
\hline
\end{tabular}

NoTE.-These are the parameters of the sinusoidal fit presented in Barnbaum \& Zuckerman 1992 to their radial velocity variations. We adjusted the phase so that it is consistent with the time base chosen here.

conclusion. Until we increase the timebase of our observations, the numbers presented here will remain only limits on what kinds of companions can be in the system.

Following the above logic, we can estimate what kind of companion could be present, yet hidden in the noise of our measurements. If we assume the long-term $(O-C)$ trend is due to something other than orbital motion, then the effect of a companion would have to be hidden in the scatter of our individual measurements. If we conservatively estimate the scatter at $\pm 10 \mathrm{~s}$ (see Fig. 4) and assume an 11.2 month period, then the minimum companion mass is $0.016 M_{\odot}$ corresponding to a mass function as described above of $9.3 \times 10^{-6} M_{\odot}$ and a radial velocity variation (assuming a circular orbit, for lack of anything better to assume) of $\pm 0.65 \mathrm{~km} \mathrm{~s}^{-1}$. Barnbaum \& Zuckerman's [1992] quoted error bars from G29-38's radial velocity measurements are between 1.5 and $2.5 \mathrm{~km} \mathrm{~s}^{-1}$.

\section{SUMMARY AND CONCLUSIONS}

We have isolated a stable peak in the transform of G29-38 in a data set spanning five observing seasons and have monitored its phase to test for the effects of a proposed orbital companion in the system. Our data are in stark disagreement with this binary model and show no compelling evidence for any coherent, long-term periodic phenomena on timescales up to 8 years. Any such periodicities would have to be contained within our scatter and would correspond to radial velocity variations that would be hidden in the uncertainty of current measurements. Our timings can be used to test any future binary models for this system - if there is a binary companion, the phase variations it would invariably produce must be consistent with the phase timings we present.
We can set limits, dependent on the chosen orbital period, for the maximum radial velocity variations that could be present, but hidden in our data. If we assume a periodicity of order one year is present, but hidden in the scatter of our data points, then a conservative estimate of the maximum resultant radial velocity variation is $\approx \pm 0.65 \mathrm{~km} \mathrm{~s}^{-1}$. Another possibility is that the long-term trend seen in our phase timings is due to orbital motion with a much longer period. Such an orbit with an $\approx 8$ yr period would produce a radial velocity variation on the order of $0.8 \mathrm{~km} \mathrm{~s}^{-1}$. We must reiterate here that these are only limits contained in our measurements; we are in no way implying the reality of any orbital variation.

There are many ways to make the phase and amplitude of a pulsation change-binary companions, nonlinear effects, secular evolution-so a unique interpretation of such changes is difficult. Stability, on the other hand, is more easily interpreted and harder to maintain.

Once again, G29-38 has continued to baffle those who study it. Asteroseismologically, we do not understand the large changes we see in its pulsations nor do we understand the phase variation we witnessed in 1988. These results present a mode that has remained stable throughout all the other changes the star has made yet does not agree with the measured radial velocity variations. The source of these too, then, remains a mystery.

The Fourier transforms were calculated at the National Center for Supercomputation (CESUP-UFRGS), Brazil, whose help we gratefully acknowledge. We thank the Louisiana State University for the loan of a two-channel photometer used in some of these observations and E. L. Robinson and Ray Steining for helping obtain some simultaneous multicolor data at McDonald. S. J. K. is grateful to the McDonald observatory for granting the large quantities of telescope time this project demanded. A. J. K. also acknowledges M. Morganfield for helpful discussions on blue filters. This work was supported in part by NSF Grants AST-8552457, AST-8600507, AST9013978, AST-9014655, AST-9217988, and National Committee for Scientific Research Grant 2-2109-91-02 (Poland).
Barnbaum, C., \& Zuckerman, B. 1992, ApJ, 396, L31

Clemens, J. C. 1994, Ph.D. thesis, Univ. Texas at Austin

Graham, J. R., Matthews, K., Neugebauer, G., \& Soifer, B. T. 1990a, ApJ, 357, 216

Graham, J. R., McCarthy, J. K., Read, I. N., \& Rich, R. M. 1990b, ApJ, 357, L21

Greenstein, J. L. 1988, AJ, 95, 1494

Kepler, S. O., et al. 1991, ApJ, 378, L45

Kleinman, S. J. 1990, M.S. thesis, Univ. of Texas at Austin

Liebert, J., Saffer, R. A., \& Pilachowski, C. A. 1989, AJ, 97, 182

McGraw, J. T., \& Robinson, E. L. 1975, ApJ, 200, 189

Nather, R. E., Winget, D. E., Clemens, J. C., Hansen, C. J., \& Hine, B. P. 1990, ApJ, 361, 309

\section{REFERENCES}

Shulov, O. S., \& Kopatskaya, E. N. 1974, Astrofizika, 10, 117

Telesco, C. M., Joy, M., \& Sisk, C. 1990, ApJ, 358, L17

Tokunaga, A. T., Becklin, E. E., \& Zuckerman, B. 1990, ApJ, 358, L21

Winget, D. E. 1988, in IAU Symp. 123, Advances in Helio- and Asteroseismology, ed. J. Christensen-Dalsgaard \& S. Frandsen (Dordrecht: Reidel), 305

- 1991, in NATO ASI Ser., Proc. 7th European Workshop on White Dwarfs, ed. G. Vauclair \& E. M. Sion (Dordrecht: Kluwer), 129 Winget, D. E., et al. 1990, ApJ, 357, 630

Zuckerman, B. 1993, in ASP Conf. Ser., Vol. 36, Planets around Pulsars, ed.

J. A. Phillips, J. E. Thorsett, \& S. R. Kulkarin (San Francisco: ASP), 303

Zuckerman, B., \& Becklin, E. E. 1987, Nature, 330, 138 